\title{
ISSN: 2539-3804 다) (하을 \\ La evaluación de los propios progresos en la expresión oral frente a la importancia concedida a los criterios de autoevaluación
}

Emma García Sanz

Universität Duisburg-Essen

Essen, Alemania

\section{Resumen}

En el aula de lenguas extranjeras, la autoevaluación se ha convertido en una parte esencial del proceso de enseñanza-aprendizaje. Este artículo tiene como objetivo analizar las similitudes y diferencias entre las autoevaluaciones semilibres hechas por discentes y sus opiniones sobre los criterios de autoevaluación (AE) empleados en el aula de expresión oral en español como lengua extranjera (ELE). Asimismo, es objeto de esta investigación reflexionar sobre los resultados obtenidos y extraer sus implicaciones para el aula de lenguas extranjeras y futuros estudios en la materia. Las conclusiones a las que llegamos reflejan, entre otros, la necesidad de formación en AE, la importancia del lenguaje empleado y la reflexión sobre el aprendizaje realizada por los alumnos. Asimismo, la importancia dada a los descriptores de la parrilla de autoevaluación no se corresponde en todos los casos con los descriptores ya aprendidos o aún por aprender.

Palabras clave: autoevaluación; español como lengua extranjera; expresión oral; investigación empírica.

\begin{abstract}
Assessment of the own progress in oral communication skills vs. the importance given to self-assessment criteria

Self-assessment has become an essential part of the teaching-learning process in foreign language classrooms. The aim of this article is to analyse the similarities and differences between student self-assessments and their own opinions about the assessment criteria provided in an oral communication skills course of Spanish as foreign language. This article also reflects on the findings and their implications for teaching and subsequent
\end{abstract}


research. Among others, the results show the necessity of teaching self-assessment, the importance of the language used and the reflection undertaken by the students. Moreover, the importance given to the qualitative aspects of spoken language use proposed by the Common European Framework of Reference for Languages does not always correspond to the descriptors which have already been learnt or which are still to be learnt.

Key words: self-assessment; Spanish as foreign language; oral communication skills; empirical research.

\section{Résumé}

L'évaluation des propres progrès en expression orale par rapport à l'importance donnée aux critères d'autoévaluation

Dans la classe de langue étrangère, l'autoévaluation est devenue une partie essentielle du processus d'enseignement-apprentissage. Le but de cet article est d'analyser les ressemblances et les différences entre les autoévaluations des apprenants et leurs opinions sur l'importance de la grille d'autoévaluation dans un cours d'expression orale en espagnol. Cet article exprime aussi des réflexions sur les implications des résultats de la recherche dans la salle de classe et pour des recherches complémentaires. Entre autres, les résultats ont montré 1) la nécessité de former les étudiants dans l'autoévaluation, 2) l'importance de la langue utilisée dans le processus et 3) la réflexion liée au processus d'autoévaluation. De plus, l'importance donnée aux descripteurs et indicateurs de la grille d'autoévaluation ne correspond pas dans tous les cas aux indicateurs qui ont été appris par les étudiants ou qui doivent encore être appris.

Mots-clés : autoévaluation; espagnol comme langue étrangère; expression orale ; recherche empirique. 


\section{Cómo CITAR ESTE ARTÍcUlO}

García, E. (2020). La evaluación de los propios progresos en la expresión oral frente a la importancia concedida a los criterios de autoevaluación. Lenguaje, 48(1), 38-59. doi: 10.25100/lenguaje.v48i1.6571 


\section{INTRODUCCIÓN}

Las teorías cognitivas y constructivistas consideran la autoevaluación (AE) como una parte integral del aprendizaje (Oscarson, 2014). Pese a las numerosas investigaciones llevadas a cabo en esta materia, aún se percibe la necesidad de realizar más estudios que permitan construir un marco teórico y una epistemología más elaborados, y de crear materiales y herramientas fundamentados a nivel teórico y basados en la investigación empírica (Oscarson, 2014).

El objetivo general de este artículo es analizar las autoevaluaciones semilibres de la expresión oral de un grupo de estudiantes de ELE tomando como referencia los criterios de autoevaluación propuestos a lo largo de la asignatura y las propias opiniones de los discentes sobre estos últimos. De esta manera examinaremos el tipo de herramienta utilizada (una evaluación semilibre basada en dos preguntas), su significación y su validez para llevar a cabo un proceso de $\mathrm{AE}$.

Empezaremos desarrollando el marco teórico en el que se enmarca el estudio empírico. A continuación, presentaremos este atendiendo a los objetivos específicos y diseño de la investigación, y expondremos y analizaremos los resultados. Por último, extraeremos las conclusiones pertinentes.

\section{MARCo TEÓRICO}

Klenowski (1995) define el concepto de AE como "the evaluation or judgment of the worth' of one's performance and the identification of one's strengths and weaknesses with a view to improving one's learning outcomes" (p. 146).

Por su parte, Boud (1995) considera que:

Self-assessment is about students developing their learning skills. It is not just another assessment technique to be set alongside others. It is about engaging learners with criteria for good practice in any given area and making complex judgements. It is not primarily about individuals giving themselves marks or grades. And it is not about supplanting the role of teachers. (p. 17).

Es decir, la AE no es solamente la evaluación de la propia actuación o la capacidad de emitir juicios sobre la propia actuación, sino que también es una forma de desarrollar estrategias de aprendizaje y mejorar los resultados de este último.

Partiendo de estas dos definiciones, a continuación expondremos el marco teórico de nuestro estudio empírico contestando a las siguientes preguntas: ¿Quién se autoevalúa? ¿Qué autoevaluarse? ¿Cuándo autoevaluarse? ¿Dónde autoevaluarse? ¿Por qué autoevaluarse? y ¿Cómo autoevaluarse? 


\section{¿Quién se autoevalúa?}

El discente, al principio guiado por el docente, lleva a cabo la AE. Muchos son los investigadores que apuestan por la formación del alumnado en esta materia ( $c f$. entre otros Blanche, 1990; Dickinson, 1987; Oscarson, 1981; Trim, 1980).

Entre los argumentos esgrimidos por estos investigadores se encuentran que la formación lleva a que el aprendiente pueda realizar juicios precisos y válidos de forma autónoma sobre la efectividad de su comunicación (Trim, 1980), ya que sin guía, el alumno no dispone de las herramientas necesarias para determinar con precisión su nivel de competencia (Leblanc y Painchaud, 1985).

Además, para Blue (1994), el estudiante necesita recordatorios constantes y guía metodológica si ha de evaluar su nivel, monitorizar su aprendizaje y analizar sus necesidades de forma efectiva.

\section{¿Qué autoevaluarse?}

Dependiendo de las necesidades del alumnado, los objetivos de aprendizaje y, en general, la significación que se le quiera otorgar a la $\mathrm{AE}$, se optará por evaluar el nivel de lengua en general, las actividades de la lengua (cf. Consejo de Europa, 2017) o, entre otros, el grado de la implicación del estudiante en el aula. A este respecto hay que tener en cuenta que es importante utilizar un lenguaje comprensible para el alumno ( $c f$. entre otros Bachman y Palmer, 1989; Blanche, 1988; Ross, 2006).

Así, una AE cuyo objetivo sea complementar la evaluación diagnóstica será diferente a aquella que forma parte de la evaluación formativa o a la que se integra en la evaluación sumativa de la asignatura.

\section{¿Cuándo autoevaluarse?}

Harris (1997) señala diferentes momentos para autoevaluarse a lo largo de un curso o asignatura. En primer lugar, se puede llevar a cabo una AE a principios de curso para que los discentes se den cuenta de que no empiezan todos con el mismo nivel. En este caso, se trata de una AE diagnóstica con cuestionarios o encuestas para hablar de experiencias de aprendizaje pasadas y reflexionar sobre los nuevos objetivos.

En segundo lugar, se pueden realizar AE a lo largo del curso coincidiendo con las evaluaciones del profesor con el fin de reflexionar sobre lo que se ha conseguido y lo que aún se ha de alcanzar. Por otra parte, una AE a mitad de curso permitirá comparar lo aprendido hasta el momento con los objetivos iniciales. Asimismo, se prestará como ayuda para redefinir estos (de forma realista).

Por último, la AE realizada a finales de curso puede ser una forma de obtener una mejor autoimagen, mejorar la autoestima y definir objetivos futuros en el aprendizaje de la lengua. 


\section{¿Dónde autoevaluarse?}

La AE puede llevarse a cabo dentro o fuera del aula. No obstante, como ya se ha señalado, el discente ha de recibir una formación específica si se quiere que este se beneficie del proceso de AE. Por ello, la integración de esta en el aula como parte del proceso de enseñanza-aprendizaje-evaluación es fundamental y no debe quedar relegada a una tarea (optativa) para casa. El tratamiento de la AE como un objetivo o una actividad de aprendizaje más permite tanto al alumnado como al profesorado sacar provecho de esta herramienta educativa.

\section{¿Por qué autoevaluarse?}

Existen muchos motivos sobre por qué la AE debe estar presente en el aula. En general, se puede decir que, entre otros, la AE ayuda a alcanzar el objetivo ideal de la educación, que es que el discente sea capaz de autodirigirse y de llevar a cabo actividades de forma independiente y responsable (Heidt, 1979). En otras palabras, la AE ayuda a desarrollar la autonomía en el aprendizaje (Fernández, 2011), ya que también aumenta la autoeficacia, la consciencia lingüística (Kissling y O'Donnell, 2015) y la consciencia del progreso individual (Harris, 1997). Igualmente, permite que los discentes puedan ver su aprendizaje en términos "personales" (Harris, 1997). En esta línea, Brown y Harris (2014) incluso llegan a proponer que no se utilice como forma de evaluación, sino como competencia esencial para promover la autorregulación.

Asimismo, en su relación con los diversos métodos de evaluación existentes, la AE no solo contribuye a variarlos, lo cual representa un factor esencial para mantener el interés y la motivación del alumno (Ross, 2006), sino que también complementa la evaluación docente, ya que algunos aspectos del aprendizaje solo pueden evaluarlos los propios discentes (Todd, 2002). Gracias al entrenamiento necesario para mejorar su eficacia, la AE lleva al mantenimiento del aprendizaje a largo plazo, lo cual es importante para el aprendizaje a lo largo de la vida (Heidt, 1979) y el fomento de la evaluación sostenible (Boud y Soler, 2016).

Por último, la AE ayuda al estudiante a darse cuenta de que aprender una lengua es diferente a aprender otras materias y que el objetivo es la comunicación más que el conocimiento sobre la lengua (Harris, 1997), aumentando la motivación y la orientación a los objetivos en el aprendizaje (Todd, 2002) y apelando a la afectividad a la hora de aprender una lengua (Oscarson, 1989). 


\section{¿Cómo autoevaluarse?}

Existen diferentes herramientas de AE. Estas podemos clasificarlas según el papel del docente y según el grado de implicación/guía del discente ${ }^{1}$.

En el primer caso, la AE puede incluir o excluir al docente. Si esta lo incluye, el profesor puede proponer una serie de herramientas al alumnado, como cuestionarios, contratos de aprendizaje o portafolios y trabajar con ellos dentro y fuera del aula. En el caso en el que la AE excluya al docente, el alumno puede desarrollar sus propias herramientas de AE como parte de su formación autónoma o el docente puede proponerle herramientas para su uso personal, como un diario de aprendizaje que solamente consulte el aprendiente.

En el segundo caso, el grado de implicación/guía del discente permite distinguir tres tipos de AE: guiada, semilibre y libre. En la AE guiada se provee al alumnado de un cuestionario, una escala de clasificación o, entre otros, de una lista de comprobación en los cuales se le invite a reflexionar. Las preguntas suelen ser cerradas o de respuesta múltiple como "Soy capaz de corregir mis errores cuando hablo".

La AE semilibre utiliza herramientas que ofrecen preguntas abiertas como ayuda para llevar a cabo la AE, como ¿En qué aspectos de la lengua he mejorado? ¿Qué tengo que mejorar en el futuro?

En la AE libre, el alumno debería ser capaz de reflexionar sobre su aprendizaje y llevar a cabo una $\mathrm{AE}$ sin guía tras un periodo de formación en $\mathrm{AE}$.

Importante sería que en el aula se combinaran las diferentes herramientas atendiendo siempre a las necesidades del grupo meta. Estas pueden variar desde las propuestas inicialmente por Oskarsson (1980) hasta las herramientas actuales más populares como las rúbricas o el portafolio (Herrera y Ferrer, 2017; Pardo, 2008).

\section{Metodología}

Esta investigación tiene los siguientes objetivos específicos. Por una parte, nos proponemos analizar qué aspectos cualitativos para la lengua hablada se consideran aprendidos durante la asignatura (o el proyecto audiovisual realizado para la evaluación sumativa de esta) y qué aspectos cualitativos para la lengua hablada aún han de mejorarse. Por otra, establecer el nivel de coherencia entre las autoevaluaciones semilibres realizadas y la importancia otorgada a los criterios de $\mathrm{AE}^{2}$, es decir, establecer si existe relación entre los criterios aprendidos o aún por aprender y la importancia concedida a los mismos.

\footnotetext{
${ }^{1}$ Téngase en cuenta que defendemos la formación del discente en materia de AE. No obstante, también creemos que este puede llevarla a cabo de forma independiente a medida que adquiere las destrezas necesarias para autoevaluarse.

2 Para ello tomaremos como referencia los resultados de esta investigación y los obtenidos en García Sanz (2018a). A estos nos referiremos como "estudio anterior".
} 
Este trabajo describe una investigación en el aula, más concretamente, un estudio de caso en el cual se hace un análisis mixto de los datos obtenidos.

\section{Participantes y contextos de la investigación}

El estudio se llevó a cabo en una universidad alemana con alumnos del Grado de Hispánicas que cursaban una asignatura de comprensión y expresión oral en ELE. En él participaron 46 estudiantes de un nivel B2+.

\section{Diseño de la investigación: instrumentos y procedimiento de recogida de datos}

En el aula de comprensión y expresión oral de ELE, que seguía una metodología comunicativa, se realizó un proyecto audiovisual como forma de evaluación sumativa ${ }^{3}$. Este consistió en la realización de tres debates y tres autoevaluaciones guiadas de estos con la parrilla de AE presentada en la Tabla 1:

Tabla 1. Parrilla de AE basada en los aspectos cualitativos del uso de la lengua hablada fragmentados: descriptores para el nivel para el nivel B2 (Consejo de Europa, 2002, pp.

32-33)

\begin{tabular}{|l|l|}
\hline \multirow{4}{*}{ Alcance } & 01. He hecho descripciones claras. \\
\cline { 2 - 3 } & 02. He buscado la palabra adecuada sin que los demás se hayan dado cuenta. \\
\cline { 2 - 3 } Cor. He utilizado oraciones complejas. \\
\hline \multirow{4}{*}{ Fluidez } & $\begin{array}{l}\text { 04. He utilizado correctamente las estructuras gramaticales aprendidas en } \\
\text { Gramática I y II }\end{array}$ \\
\cline { 2 - 3 } & 05. Los errores gramaticales que he cometido han provocado la incomprensión. \\
\cline { 2 - 3 } & 06. He corregido los errores gramaticales que he cometido. \\
\hline \multirow{3}{*}{ Interacción } & 07. El ritmo en mi discurso ha sido uniforme. \\
\cline { 2 - 3 } & 09. He dudado buscando estructuras o expresiones. \\
\cline { 2 - 3 } & 10. He sido capaz de iniciar y terminar el discurso. \\
\cline { 2 - 3 } & 11. He sido capaz de tomar el turno de palabra en el momento adecuado. \\
\hline
\end{tabular}

${ }_{3}$ Cf. García Sanz (2018b) sobre los aspectos académicos, de aprendizaje, organizativos y sociales del proyecto audiovisual.

${ }^{4}$ Con el objetivo de que los alumnos pudieran evaluar mejor su nivel gramatical, se nombraron las dos asignaturas de gramática de nivel B2 que habían cursado en la misma universidad durante el primer y el segundo semestre y no la descripción presentada por el MCER "control gramatical relativamente alto" (Consejo de Europa, 2002, p. 32). 


\begin{tabular}{|l|l|}
\hline \multirow{3}{*}{ Coherencia } & 12. He comprendido el debate, he invitado a los otros a participar. \\
\cline { 2 - 2 } & 13. He utilizado mecanismos de cohesión. \\
\cline { 2 - 3 } & 14. Mi discurso ha sido claro. Se ha entendido lo que quería decir. \\
\cline { 2 - 3 } & 15. Me huesto nervioso cuando he tenido que hablar mucho tiempo. \\
\hline
\end{tabular}

Asimismo, los estudiantes hicieron una reflexión sobre el proyecto audiovisual en la que 1) expresaron su opinión sobre la importancia de los diferentes descriptores propuestos en la parrilla de $\mathrm{AE}^{5}$; 2) realizaron una $\mathrm{AE}$ semilibre, para lo que evaluaron su nivel de lengua después de realizar los tres debates atendiendo a lo que habían aprendido y lo que tenían que mejorar tomando como referencia la parrilla de AE de la Tabla 1 y 3) analizaron y comentaron el proyecto.

El instrumento para llevar a cabo este estudio es, pues, la reflexión sobre el proyecto audiovisual realizada al finalizar la asignatura. Más específicamente, se analizarán la valoración de los descriptores propuestos en la Tabla 1 y la AE semilibre realizada con base en dos preguntas: ¿Qué aspectos he mejorado? y ¿Qué aspectos he de mejorar en mi semestre de Erasmus?

Los descriptores finales tenidos en cuenta en el análisis son los ya presentados en la Tabla 1 (1-15) y los hallados en las autoevaluaciones semilibres realizadas por los alumnos. Estos últimos son:

- 16. Creo que es mejor hacer debates sin apuntes.

- 17. Hablo de forma espontánea, libre, sin pensar.

- 18. Estructuro mejor los documentos.

- 19. Creo que ya no pasa nada si se cometen errores.

- 20. He ganado en autoconfianza / seguridad.

- 21. He ampliado mi vocabulario.

- 22. He mejorado la comprensión auditiva.

- 23. Soy capaz de reaccionar ante lo que dicen los demás.

- 24. He mejorado la gramática.

- 25. He adquirido estrategias de vocabulario, como hacer circunloquios o usar sinónimos.

- 26. Sé cómo hacer un debate en español.

- 27. Tengo que aprender frases hechas.

- 28. Tengo que aprender a mantener conversaciones sobre temas cotidianos.

- 29. Tengo que mejorar la pronunciación.

- 30. Tengo que aprender un registro más culto.

${ }^{5}$ Cf. García Sanz (2018a) sobre la importancia para el alumnado de los diferentes descriptores formulados en la parrilla de autoevaluación. 
- 31. Tengo que mejorar la comprensión oral.

- 32. Tengo que mejorar la expresión oral en general.

Los descriptores 16-26 corresponden a los hallados en respuesta a la pregunta “¿qué he mejorado?"; los descriptores 27-32, a los encontrados en respuesta a los aspectos que se han de mejorar durante el semestre Erasmus.

\section{Resultados}

Los datos obtenidos se analizaron de forma mixta. Se utilizó la estadística descriptiva para analizar las características generales de las autoevaluaciones entregadas: medias y desviaciones típicas, tablas de contingencia y análisis de correlación.

En primer lugar, expondremos una serie de características generales de las autoevaluaciones realizadas:

- De los 46 participantes en el estudio, tres (el 6,5\%) no llevan a cabo una AE de la lengua propiamente dicha, sino que se limitan a analizar (los problemas presentados en) los debates.

- En el análisis de las autoevaluaciones se tomó como primera referencia la parrilla de AE propuesta a lo largo del semestre para los debates realizados. A los descriptores propuestos se añadieron aquellos diferentes mencionados por los estudiantes. A la hora de llevar a cabo la AE final, el $7 \%$ de los discentes se basó en la parrilla de $\mathrm{AE}$ enumerando los criterios en los que consideraba que había mejorado. El 32,6 \% realizó una AE "libre" en la que no se refirió textualmente a estos. El 60,5\% restante utilizó los criterios trabajados a lo largo del semestre e incluyó otros propios o los mismos reformulados con sus propias palabras.

- La longitud media de las AE es de 169 palabras, teniendo la más corta 46 palabras y la más larga 370. La desviación estándar es de s' =67,646 (cf. la Figura 1). 


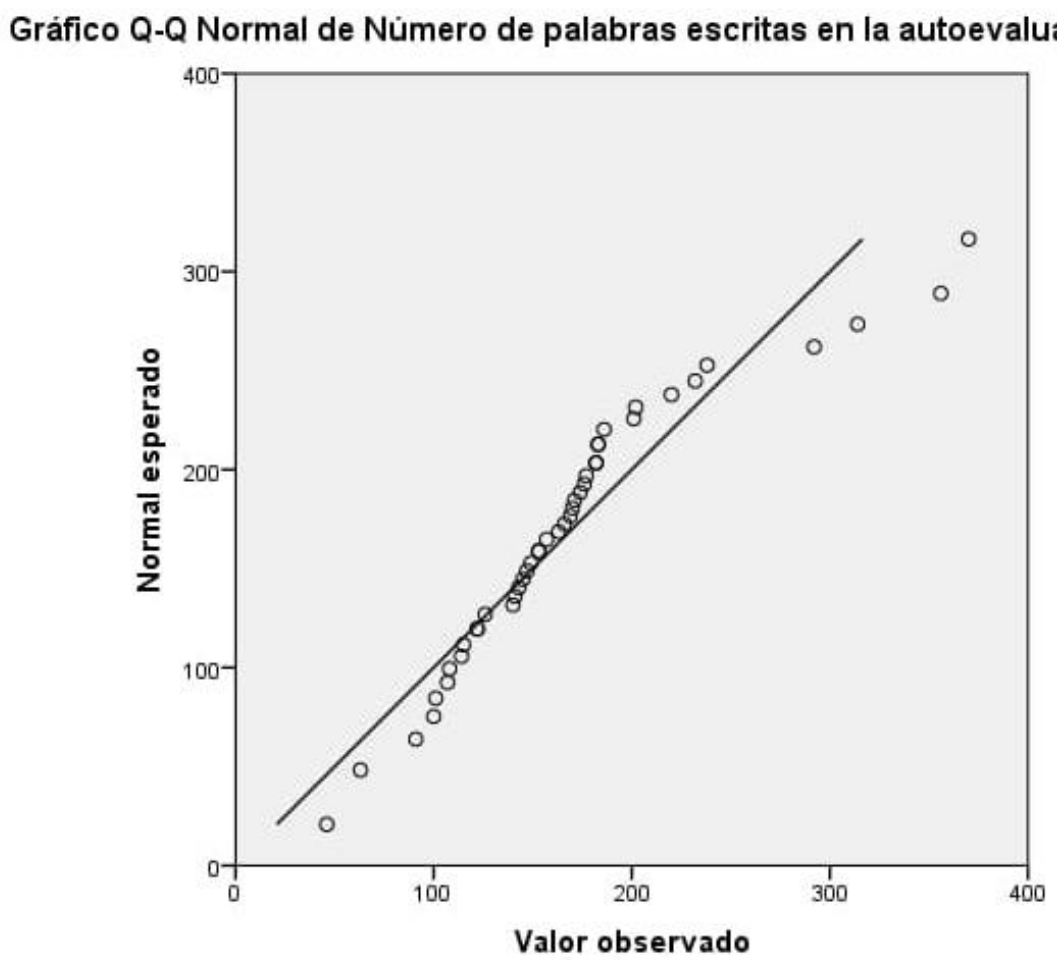

Figura 1. Gráfico Q-Q Normal de Número de palabras de la AE

- El número medio de los aspectos mencionados (aprendidos y por mejorar) es 5, siendo el mínimo 2 y el máximo 11. La desviación estándar es de s" $=2,2$.

- El número medio de los aspectos aprendidos es 3, siendo el mínimo 1 y el máximo 6. La desviación estándar es de $\mathrm{s}^{\prime \prime \prime}=1,5$.

- El número medio de los aspectos por mejorar es 2, siendo el mínimo 0 y el máximo 6. La desviación estándar es de s'"' $=1,2$. El 11,6 \% de los estudiantes no menciona los aspectos que ha de mejorar.

- No existe correlación significativa $(\alpha=, 01)$ entre el número de palabras escritas en la $\mathrm{AE}$ y el número de los descriptores aprendidos o por mejorar.

En segundo lugar, analizaremos los aspectos en los que los estudiantes consideraron que habían mejorado. Además de los descriptores mencionados anteriormente en la parrilla de AE ( $c f$. Tabla 1), en el análisis de las autoevaluaciones también se encontraron los descriptores 16-26 presentados anteriormente.

Entre los descriptores de mayor éxito en el aprendizaje se encuentran el 17, el 21 y el 15. El descriptor 17, "Hablar de forma espontánea, libre, sin pensar", representa el descriptor que más alumnos mencionan como aprendido (el 55,8\%). Le sigue el descriptor 21, "He ampliado mi vocabulario.", elegido por el 41,9\%. El tercer lugar lo ocupa el descriptor 15, "Me he puesto nervioso cuando he tenido que hablar mucho tiempo.", seleccionado por el 39,5\%. 
En la Tabla 2 se presentan todos los porcentajes de estudiantes que mencionan los descriptores anteriores como aprendidos a lo largo del semestre.

Tabla 2. Descriptores mencionados como aprendido en \%

\begin{tabular}{|c|c|}
\hline Descriptor mencionado & $\begin{array}{l}\text { Porcentaje } \\
\text { de alumnos }\end{array}$ \\
\hline 17. Hablo de forma espontánea, libre, sin pensar. & 55,8 \\
\hline 21. He ampliado mi vocabulario. & 41,9 \\
\hline 15. Me he puesto nervioso cuando he tenido que hablar mucho tiempo. & 39,5 \\
\hline 16. Creo que es mejor hacer debates sin apuntes. & 25,6 \\
\hline 01. He hecho descripciones claras. & 16,3 \\
\hline 10. He sido capaz de iniciar y terminar el discurso. & 16,3 \\
\hline 11. He sido capaz de tomar el turno de palabra en el momento adecuado. & 11,6 \\
\hline 13. He utilizado mecanismos de cohesión. & 11,6 \\
\hline 20. He ganado en autoconfianza / seguridad. & 11,6 \\
\hline 03. He utilizado oraciones complejas. & 9,3 \\
\hline 24. He mejorado la gramática. & 9,3 \\
\hline 06. He corregido los errores gramaticales que he cometido. & 7 \\
\hline $\begin{array}{l}\text { 25. He adquirido estrategias de vocabulario, como hacer circunloquios o } \\
\text { usar sinónimos. }\end{array}$ & 7 \\
\hline 26. Sé cómo hacer un debate en español. & 7 \\
\hline 07. El ritmo de mi discurso ha sido uniforme. & 4,7 \\
\hline 08. He realizado pausas largas. & 4,7 \\
\hline 09. He dudado buscando estructuras o expresiones. & 4,7 \\
\hline 12. He comprendido el debate, he invitado a los otros a participar. & 4,7 \\
\hline 19. Creo que ya no pasa nada si se cometen errores. & 4,7 \\
\hline 22. He mejorado la comprensión auditiva. & 4,7 \\
\hline $\begin{array}{l}\text { 02. He buscado la palabra adecuada sin que los demás se hayan dado } \\
\text { cuenta. }\end{array}$ & 2,3 \\
\hline 14. Mi discurso ha sido claro. Se ha entendido lo que quería decir. & 2,3 \\
\hline 18. Estructuro mejor los documentos. & 2,3 \\
\hline 23. Soy capaz de reaccionar ante lo que dicen los demás. & 2,3 \\
\hline $\begin{array}{l}\text { 04. He utilizado correctamente las estructuras aprendidas en Gramática I y } \\
\text { II. }\end{array}$ & 0 \\
\hline $\begin{array}{l}\text { 05. Los errores gramaticales que he cometido han provocado la } \\
\text { incomprensión. }\end{array}$ & 0 \\
\hline
\end{tabular}

Asimismo, hallamos las correlaciones de cada descriptor aprendido con la importancia otorgada al mismo. Solamente en el caso de los descriptores 6, 10 y 13 existe correlación entre el descriptor aprendido y la importancia concedida al mismo ( $\varrho 6$ = ,470; 
$\mathrm{Q} 10=, 430 ; \mathrm{Q} 13=, 482$ respectivamente) para un $\alpha=, 01$. En estos tres casos, el porcentaje de alumnos que no los considera descriptores aprendidos ni importantes es del $86 \%$, $79,1 \%$ y $81,4 \%$ respectivamente.

Para completar el análisis de los descriptores, los agrupamos según su pertenencia a los aspectos cualitativos del uso de la lengua hablada propuestos en el MCER (Consejo de Europa, 2002) utilizados en la parrilla de AE, añadiendo un sexto con aquellos aspectos imposibles de clasificar en los cinco primeros:

- Alcance $(1,2,3,21,25)$

- Corrección $(4,5,6,24)$

- Fluidez $(7,8,9,17)$

- Interacción $(10,11,12,22,23)$

- Coherencia $(13,14,15)$

- $\operatorname{Otros}(16,18,19,20,26)$

En la Tabla 3 se muestran los porcentajes de estudiantes que consideran los aspectos anteriores aprendidos.

Tabla 3. Aspectos cualitativos de la lengua hablada aprendidos en $\%$

\begin{tabular}{|l|l|l|l|l|l|l|}
\hline & Alcance & Fluidez & Coherencia & Otros & Interacción & Corrección \\
\hline Aprendidos & 60,5 & 60,5 & 48,8 & 41,9 & 30,2 & 16,3 \\
\hline
\end{tabular}

Con el fin de analizar las relaciones entre los propios aspectos cualitativos aprendidos, hallamos las tablas de contingencia correspondientes y llevamos a cabo un análisis de correlación entre ellos. Las pruebas estadísticas no reportan correlaciones significativas entre los aspectos cualitativos aprendidos. Además, calculamos las correlaciones entre estos y los aspectos cualitativos más y menos importantes analizados en el estudio anterior. Estas tampoco son estadísticamente significativas. No obstante, las tablas de contingencia obtenidas revelan los siguientes aspectos interesantes para la investigación:

- El 51,15\% cree que el alcance es un aspecto cualitativo importante y que lo ha aprendido.

- El 37,21\% considera la fluidez como un aspecto cualitativo no importante pero aprendido.

- El 34,89\% afirma que la coherencia es importante y que la ha aprendido.

En tercer lugar, los discentes estimaron que tenían que mejorar en algunos de los descriptores mencionados anteriormente y en los siguientes nuevos:

Entre los descriptores que se consideran mayoritariamente como pendientes de mejorar, se encuentran en primer lugar, con un 41,9\% de estudiantes que los eligen, el 21 “Tengo que ampliar mi vocabulario" y el 24 "Tengo que mejorar la gramática". El $34 \%$ 
considera que tiene que practicar el descriptor 17: "Tengo que practicar el hablar de forma espontánea, libre, sin pensar"6.

Los porcentajes de alumnos que reportan que han de progresar en los descriptores anteriores pueden consultarse en la Tabla 4:

Tabla 4. Descriptores mencionados como pendientes de mejorar en \%

\begin{tabular}{|l|l|}
\hline \multicolumn{1}{|c|}{ Descriptor mencionado } & $\begin{array}{l}\text { Porcentaje } \\
\text { de alumnos }\end{array}$ \\
\hline 21. Tengo que ampliar mi vocabulario. & 41,9 \\
\hline 24. Tengo que mejorar la gramática. & 41,9 \\
\hline $\begin{array}{l}\text { 17. Tengo que practicar el hablar de forma espontánea, libre, sin pensar. } \\
\text { 29. Tengo que mejorar la pronunciación. }\end{array}$ & 34,9 \\
\hline $\begin{array}{l}\text { 15. Tengo que evitar ponerme nervioso cuando tenga que hablar mucho } \\
\text { tiempo. }\end{array}$ & 16,3 \\
\hline 32. Tengo que mejorar la expresión oral en general. & 14 \\
\hline 11. Tengo que mejorar cuándo tomar el turno de palabra. & 11,6 \\
\hline 13. Tengo que utilizar mecanismos de cohesión. & 7 \\
\hline 01. Tengo que hacer descripciones más claras. & 7 \\
\hline 27. Tengo que aprender frases hechas. & 2,3 \\
\hline $\begin{array}{l}\text { 28. Tengo que aprender a mantener conversaciones sobre temas } \\
\text { cotidianos. }\end{array}$ & 2,3 \\
\hline 30. Tengo que aprender un registro más culto. & 2,3 \\
\hline 31. Tengo que mejorar la comprensión oral. & 2,3 \\
\hline
\end{tabular}

Además, hallamos las correlaciones de cada descriptor aprendido con la importancia otorgada al mismo sin encontrar significación estadística.

Para un mejor análisis de dichos descriptores, los agrupamos según su pertenencia a los aspectos cualitativos del uso de la lengua hablada propuestos en el MCER (Consejo de Europa, 2002) y que habíamos utilizado para la parrilla de AE:

- Alcance (1, 21, 27, 28 y 30)

- Corrección (24)

- Fluidez (17)

- Interacción (11 y 31)

- Coherencia (13 y 15)

El descriptor 29, la pronunciación, lo consideramos dentro del sexto aspecto cualitativo propuesto en Consejo de Europa (2017).

${ }^{6}$ Los descriptores $1,13,15,17,21$ y 24 se han reformulado adaptándolos al objetivo específico de la autoevaluación, es decir, reflexionar sobre los aspectos que se han de mejorar y no los aspectos aprendidos. 
- Fonología (29)

Respecto a la expresión oral en general (descriptor 32), decidimos no incluirla en ningún aspecto cualitativo. A este respecto, en general, el 11,6\% considera que ha de mejorarla frente al $88,4 \%$ que no la menciona.

Los resultados obtenidos se presentan en la Tabla 5:

Tabla 5. Aspectos cualitativos de la lengua hablada por mejorar en $\%$

\begin{tabular}{|l|r|r|r|r|r|r|}
\hline & \multicolumn{1}{|c|}{ Alcance } & Corrección & Fluidez & Coherencia & Fonología. & Interacción \\
\hline Por mejorar & 51,2 & 41,9 & 34,9 & 20,9 & 16,3 & 7 \\
\hline
\end{tabular}

Por último, hallamos las tablas de contingencia y las correlaciones entre los propios aspectos cualitativos por mejorar y entre los aspectos cualitativos por mejorar y los aspectos cualitativos más y menos importantes. No obtuvimos ninguna correlación significativa. Las tablas de contingencia reportan los siguientes resultados interesantes:

- El 44,19\% de los estudiantes considera el alcance un aspecto importante y por mejorar.

- El $41,85 \%$ de los aprendientes señala la fluidez como aspecto cualitativo no importante, pero también piensa que ha de mejorarlo.

- El 32,56 \% de los alumnos cree que la corrección es importante y ha de mejorarla.

Asimismo, las correlaciones halladas entre los aspectos cualitativos aprendidos y los aspectos cualitativos por mejorar no son estadísticamente significativas.

\section{Interpretación de los resultados}

Primero, recordemos que Leblanc y Painchaud (1985) argumentaban que si se deja al alumnado hacer una $\mathrm{AE}$ con sus propias fuentes, este no dispone de las herramientas necesarias para determinar con precisión su nivel de competencia. Asimismo, numerosos investigadores apuestan por la formación del estudiantado en materia de AE (cf. entre otros Blanche, 1990; Dickinson, 1987; Oscarson, 1981; Trim, 1980).

En nuestro caso, los estudiantes no tenían que precisar el nivel alcanzado durante el semestre, sino reflexionar sobre los aspectos que habían aprendido y los que tenían que mejorar. Sin embargo, el hecho de que el 6,5\% de los alumnos no fuera capaz de realizar la AE pese a haber estado trabajando sobre ella durante toda la asignatura refuerza la necesidad de la formación y de aceptar que el alumnado necesita recordatorios constantes

\footnotetext{
7 A este respecto consideramos importante y necesario actualizar la parrilla de evaluación propuesta en García Sanz (2018a) con el aspecto cualitativo de la fonología siguiendo las directrices expuestas en Consejo de Europa (2017).
} 
y guía metodológica si ha de evaluar su nivel, monitorizar su aprendizaje y analizar sus necesidades de forma efectiva (Blue, 1994).

Segundo, respecto al alumnado que sí realizó la AE podemos destacar la importancia del lenguaje utilizado. Solamente el $7 \%$ utiliza los descriptores pertenecientes a la parrilla de AE con la que había trabajado a lo largo del semestre. El $32,6 \%$ utiliza términos exclusivamente propios y el $60,5 \%$ se vale de los descriptores dados y añade otros nuevos. Así, en el caso de los descriptores aprendidos, la mitad pertenece a un aspecto cualitativo del MCER. De los descriptores que se han de mejorar, en el $69,23 \%$ de los casos se hace uso de las propias palabras. De estos hechos concluimos que 1) el lenguaje utilizado en la $\mathrm{AE}$ es clave en el éxito de la misma (al respecto, varios autores han señalado la importancia de este en las autoevaluaciones a la hora de analizar su fiabilidad, $c f$. entre otros Blanche, 1988; Ross, 2006). 2) El hecho de que los discentes creen nuevos descriptores que no sean una mera reformulación de los propuestos en la parrilla de AE sugiere que la formación y los momentos de reflexión llevados a cabo durante el semestre ayudaron a los aprendientes a hacer uso de y completar las herramientas necesarias para autoevaluarse.

Tercero, en cuanto al número de palabras escritas en la $\mathrm{AE}$ cabe señalar que aunque se aprecian diferencias considerables entre la AE más corta (46 palabras) y la más larga (370 palabras), en general la mayoría se sitúa entre 100 y 200 palabras. La mayor longitud de la AE no implica necesariamente que los alumnos expongan más descriptores aprendidos o por mejorar (recordemos que el coeficiente de correlación de Pearson para un $\alpha=, 01$ no es significativo). Esto se debe a la forma de redacción de la AE, siendo así más o menos concisa o, en otras palabras, teniendo mayores o menores circunloquios. Así, este es un factor a tener en cuenta de cara a futuras evaluaciones con la misma herramienta en las que quizá más que establecer un número determinado de palabras ${ }^{8}$, se podría establecer un número de observaciones sobre el progreso.

Cuarto, se mencionan de media más descriptores aprendidos que por mejorar y el $11,6 \%$ no hace referencia a los últimos pese a que en la guía para llevar a cabo la AE se señalaban como un aspecto sobre el cual reflexionar. Esto puede ser influencia del tipo de AE llevado a cabo durante el semestre, en el cual los discentes autoevaluaron los debates. Dado que en esos momentos la docente no pidió una reflexión sobre los aspectos por mejorar, no es seguro que todos los estudiantes analizaran los mismos de cara a preparar las siguientes clases o los próximos debates. Es más, en muchos casos se pidió más evaluación por parte de la docente para saber en qué aspectos habían de progresar (cf. García Sanz, 2018b). A este respecto, volvemos a hacer referencia a las palabras de Blue (1994) sobre la necesidad de guiar al discente en el proceso.

\footnotetext{
${ }^{8}$ Aunque en este caso no se estableció un número determinado de palabras para la autoevaluación en particular, sí se hizo para la reflexión sobre el proyecto audiovisual en el cual se enmarcaba.
} 
Quinto 9 , en cuanto a los aspectos cualitativos en general, más de la mitad de los estudiantes, el 60,5\%, admite que ha mejorado en el alcance. Este resultado se corresponde con la importancia que los discentes otorgaban a este aspecto, habiéndolo situado como el más importante a la hora de realizar una AE. En cuanto a los descriptores pertenecientes a este aspecto, el estudiantado (un 41,9\%) estima que el 21, “He ampliado mi vocabulario", es el segundo descriptor en el que ha avanzado.

En el mismo lugar se encuentra la fluidez, considerada también por el 60,5\% como aprendida. El 55,8 \% piensa que ha mejorado a la hora de "hablar de forma espontánea, libre, sin pensar" (descriptor 17). El hecho de que los demás descriptores correspondientes a este aspecto cualitativo apenas estén o no representados en las autoevaluaciones, se corresponde con los resultados obtenidos en el estudio anterior, en el que la fluidez quedaba relegada a la última posición en importancia según los descriptores señalados como importantes, pero en el aula se consideraba ("su idea de fluidez") como un aspecto fundamental.

La coherencia es el único aspecto en el que solamente se exponen los descriptores dados para analizar los conocimientos aprendidos. Cabe volver a mencionar la contradicción en los resultados obtenidos en el descriptor 15, "Me he puesto nervioso cuando he tenido que hablar mucho tiempo". A este descriptor (junto al descriptor 8) le otorgaba el 56,5\% del alumnado la menor importancia en el aprendizaje. No obstante, del 39,5\% que consideraba que había mejorado el nerviosismo en su expresión oral, el $25,57 \%$ señalaba que este descriptor era uno de los menos importantes de la parrilla de AE. Esto nos lleva a poder constatar que los resultados de la AE también pueden depender del lenguaje y la herramienta utilizados.

El 30,2 \% de los alumnos señala que ha mejorado en la interacción. En cuanto a la importancia otorgada en el estudio anterior, este aspecto cualitativo ocupaba un lugar neutro.

La corrección ocupa el último lugar, habiendo sido elegida por el 16,3\% del alumnado como aspecto aprendido. La relevancia que tenía en el estudio anterior, ocupando el segundo puesto en significación, no se corresponde con la posición obtenida en el aprendizaje. Esto constituye el ejemplo contrario a los resultados obtenidos con el descriptor 15.

En general, la ausencia de correlaciones entre los descriptores aprendidos y los descriptores importantes nos lleva a afirmar que no siempre que se considera un descriptor importante, también es considerado aprendido, como es el caso más notable de los descriptores pertenecientes a la corrección. Asimismo, también se puede afirmar que no siempre que se valora un descriptor como no importante, no se considera aprendido (cf. el descriptor 15).

\footnotetext{
9 A continuación llevaremos a cabo la interpretación de los resultados comparándolos con aquellos obtenidos en García Sanz (2018a).
} 
Sexto, en cuanto a los aspectos cualitativos por mejorar, el alcance ocupa el primer lugar con un 51,2 \% del alumnado que lo menciona. Esta posición se corresponde con los resultados hallados en el estudio anterior, en el cual el alcance se consideraba el aspecto cualitativo más importante. No obstante, es necesario señalar que la primera posición se debe, sobre todo, al descriptor 21 "Tengo que ampliar mi vocabulario". De esta forma, los estudiantes vuelven a demostrar la transcendencia del tipo de lenguaje (comprensible) utilizado en la AE.

La corrección es seleccionada por el 41,9\% del estudiantado, situándose en segundo lugar. En este caso se observa el mismo fenómeno que en el alcance. Los alumnos vuelven a hacer uso de su propio lenguaje considerando importante la "gramática" y no los descriptores de la parrilla de AE.

La fluidez aparece en tercer lugar, seleccionada por el 34,9\%, debiéndose este puesto al descriptor 17, "Tengo que practicar el hablar de forma espontánea, libre, sin pensar". En el estudio anterior se llamó la atención sobre la idea errónea de los estudiantes del aspecto cualitativo de la fluidez, ya que en el aula dieron la definición de esta como simplemente "hablar deprisa". En la AE aparece definida como hablar de forma espontánea, libre, "sin pensar".

La coherencia la considera un $20,9 \%$ del alumnado como un aspecto cualitativo por mejorar. Este porcentaje es debido, mayoritariamente, al descriptor 15. La interacción, por su parte, ocupa el último lugar. En el estudio anterior no destacaba por su importancia (mayor o menor).

Séptimo y último, de todos los aspectos cualitativos mencionados destacan el alcance y la fluidez como los dos aspectos en los que el alumnado ha progresado y aún considera que tiene que mejorar. El alcance era, además, el aspecto cualitativo más importante para los estudiantes. Con ello se vuelve a constatar la relevancia en general que se le concede. Y es que sin vocabulario no se puede comunicar nada (Wilkins, 1972) ${ }^{10}$. Por el contrario, pese a que la fluidez (su concepción) era considerada como el aspecto menos relevante, su significación en la evaluación de la propia lengua tiene gran relevancia tanto como aspecto aprendido como aspecto por mejorar. Coherentes son las posiciones que ocupa la corrección, del aspecto menos aprendido, al segundo en importancia a la hora de mejorar la competencia comunicativa. En cuanto a la coherencia, cabe destacar el papel del descriptor 15, como ya se ha mencionado anteriormente. Puede considerarse que el lugar neutro ocupado por la interacción en el estudio anterior es el mismo que en este estudio, ya que se sitúa entre los aspectos que no se mencionan ampliamente como mejorados o por mejorar.

10 "Without grammar little can be conveyed; without vocabulary nothing can be conveyed" (Wilkins, 1972, p. 111). 


\section{CONCLUSIONES}

Teniendo en cuenta las limitaciones de esta investigación en cuanto al número de participantes (46) y el contexto educativo (universidad), podemos formular las siguientes conclusiones derivadas de la interpretación de los resultados.

En primer lugar, el análisis nos lleva a poder corroborar diferentes afirmaciones en el campo de la AE, como son la necesidad de guiar al alumno en la realización de la AE para que esta sea efectiva, la importancia del lenguaje utilizado (los estudiantes no solamente utilizan descriptores conocidos, sino que los reformulan con sus propias palabas y crean otros nuevos) y la importancia de la herramienta elegida (en una $\mathrm{AE}$ semilibre los resultados obtenidos pueden diferir sustancialmente de aquellos hallados en una evaluación guiada).

En segundo lugar, debido a la defensa de la AE como herramienta para fomentar la autorregulación y partiendo de que el aprendizaje de la lengua no solamente se llevaba a cabo en el aula, se podría esperar una correlación entre la importancia que se concede a los descriptores y los descriptores realmente aprendidos o que aún se han de aprender. Sin embargo, esto no se corresponde con los resultados. Esto es, 1) que un descriptor se considere importante no quiere decir que se considere aprendido o aún se tenga que aprender y 2) que un descriptor no se considere importante no quiere decir expresamente que no se haya aprendido o que no se tenga que profundizar en él (salvo en el caso de los descriptores 6, 10 y 13, considerados no importantes y no aprendidos). Igualmente, el análisis de los aspectos cualitativos tampoco presenta una correlación entre la importancia concedida a cada uno de ellos y los que se han aprendido o aún han de mejorarse. No obstante, cabe destacar la posición del alcance, que se considera el aspecto cualitativo más importante, en el que más se ha progresado y, paradójicamente, también el que mayor número de estudiantes considera que ha de desarrollar. De esta forma, si la importancia otorgada a los aspectos cualitativos no se considera un parámetro para decidir qué se ha de acrecentar, esto nos lleva a plantearnos la necesidad de investigar sobre las posibles características o circunstancias que influyen en el aprendiente a la hora de planificar su aprendizaje luego de realizar una AE (téngase en cuenta las definiciones de AE presentadas al principio del trabajo).

Asimismo, la investigación nos hace abordar una serie de cuestiones para futuros procesos de AE. Por una parte, sería fundamental plantearse la reformulación por parte de los estudiantes de los descriptores de la AE con base en los dados. Con ello se aseguraría un lenguaje comprensible para todos los estudiantes y de mayor facilidad de uso. Por otra parte, en caso de elegir una AE semi-libre, sería importante reflexionar sobre si se les pide a los alumnos una $\mathrm{AE}$ de un número determinado de palabras o se les pide un número específico de aspectos (descriptores) por evaluar, ya que, como demuestran los resultados, un mayor número de palabras no demuestra necesariamente un mayor número de aspectos por evaluar. 
Por último, cabe destacar la importancia de establecer futuras líneas de investigación en materia de $\mathrm{AE}$ en cuanto a las herramientas utilizadas. En nuestra investigación, los estudiantes redactaron su $\mathrm{AE}$ semilibre a partir de la propuesta de dos preguntas (¿Qué aspectos he mejorado? ¿Qué aspectos he de mejorar en mi semestre de Erasmus?). Sería interesante investigar los resultados que podrían obtenerse si en vez de realizar la autoevaluación en forma de redacción, los alumnos se autoevaluaran utilizando, por ejemplo, un cuestionario, un portafolio o un contrato de aprendizaje o qué herramienta sería más útil dependiendo de los contenidos por evaluar.

\section{REFERENCIAS}

Bachman, L. F., y Palmer, A. S. (1989). The construct validation of self-ratings of communicative language ability. Language Testing, 6(1), 14-29. doi: 10.1177/026553228900600104.

Blanche, P. (1988). Self-Assessment of Foreign Language Skills: Implications for Teachers and Researchers. RELC Journal, 19(1), 75-93. doi: 10.1177/003368828801900105.

Blanche, P. (1990). Using standardized achievement and oral proficiency tests for selfassessment purposes: the DLIFLC study. Language Testing, 7(2), 202-229. doi: $10.1177 / 026553229000700205$.

Blue, G. M. (1994). Self-Assessment of Foreign Language Skills: Does It Work? CLE Working Papers, (3), 18-35.

Boud, D. (1995). Enhancing Learning through Self-Assessment. London: Kogan Page.

Boud, D., y Soler, R. (2016). Sustainable assessment revisited. Assessment \& Evaluation in Higher Education, 41(3). doi: 10.1080/02602938.2015.1018133.

Brown, G. T. L., y Harris, L. R. (2014). The future of self-assessment in classroom practice: Reframing self-assessment as a core competency. Frontline Learning Research, 2(1), 22-30. doi: 10.14786/flr.v2i1.24.

Consejo de Europa. (2002). Marco Común Europeo de referencia para las lenguas: aprendizaje, enseñanza, evaluación. Recuperado de https://cvc.cervantes.es/ensenanza/biblioteca_ele/marco/.

Consejo de Europa. (2017). Common European Framework of Reference for Languages: Learning, Teaching, Assessment. Companion Volume with new Descriptors. Estrasburgo: Council of Europe Publishing.

Dickinson, L. (1987). Self-instruction in Language Learning. Cambridge: Cambridge University Press.

Fernández, S. (2011). La autoevaluación como estrategia de aprendizaje. MarcoELE, (13). Recuperado de https://marcoele.com/descargas/13/sonsolesfernandez_autoevaluacion.pdf.

García Sanz, E. (2018a). La autoevaluación en la comprensión y expresión oral: análisis de sus criterios desde la perspectiva del discente. MarcoELE, Revista electrónica de 
didáctica-español lengua extranjera, (26). Recuperado de https://marcoele.com/autoevaluacion-en-la-comprension-y-expresion-oral/.

García Sanz, E. (2018b). “@debate“: Análisis de un proyecto audiovisual en el aula de ELE.

DIGILEC: Revista Internacional de Lenguas y Culturas, 5, 70-83. doi: 10.17979/digilec.2018.5.0.3495.

Harris, M. (1997). Self-assessment of language learning in formal settings. ELT Journal, 51(1), 12-20. doi: 10.1093/elt/51.1.12.

Heidt, E. U. (1979). Self-evaluation in learning: A report on trends, experiences and research findings. París: UNESCO, Division of Structures, Content, Methods, and Techniques of Education. Recuperado de http://unesdoc.unesco.org/images/0003/000362/036216eb.pdf.

Herrera, J., y Ferrer, À. (2017). Evaluación y autorregulación del aprendizaje: la experiencia del Instituto Cervantes de Belgrado. Presentado en el III Congreso Internacional SICELE. Investigación e innovación en ELE. Evaluación y variedad lingüística del español. Recuperado de http://cvc.cervantes.es/ensenanza/biblioteca_ele/sicele/011_herreraferrer.htm.

Kissling, E. M., y O’Donnell, M. E. (2015). Increasing language awareness and self-efficacy of FL students using self-assessment and the ACTFL proficiency guidelines. Language Awareness, 24(4), 283-302. doi: 10.1080/09658416.2015.1099659.

Klenowski, V. (1995). Student Self-evaluation Processes in Student-centred Teaching and Learning Contexts of Australia and England. Assessment in Education, 2(2), 145163. doi: 10.1080/0969594950020203.

LeBlanc, R., y Painchaud, G. (1985). Self-Assessment as a Second Language Placement Instrument. TESOL Quarterly, 19(4), 673-687. doi: 10.2307/3586670.

Oscarson, M. (1981). L'auto-évaluation dans l'apprentissage des langues pour adultes: Quelques résultats de recherches. Études de Linguistique Appliquée, 41, 102-115.

Oscarson, M. (1989). Self-assessment of language proficiency: rationale and applications. Language Testing, 6(1), 1-13. doi: 10.1177/026553228900600103.

Oscarson, M. (2014). Self-Assessment in the Classroom. En A. Kunnan (Ed.), The Companion to Language Assessment (Vol. 2) (pp. 712-729). doi: 10.1002/9781118411360.wbcla046.

Oskarsson, M. (1980). Approaches to Self-assessment in Foreign Language Learning. Oxford y Nueva York: Pergamon Press.

Pardo, J. (2008). La autoevaluación y la coevaluación en una enseñanza centrada en la práctica reflexiva. Recuperado de http://www.educacionyfp.gob.es/dam/jcr:e2811249-379f-4920-ad1009841b441222/2011-bv-12-13pardo-pdf.pdf.

Ross, J. A. (2006). The Reliability, Validity, and Utility of Self-Assessment. Practical Assessment, Research \& Evaluation, 11(10). Recuperado de 
https://pareonline.net/getvn.asp? $\mathrm{v}=11 \& \mathrm{n}=10$.

Todd, R. (2002). Using Self-assessment for Evaluation. English Teaching Forum, 40(1), 1619. Recuperado de https://americanenglish.state.gov/files/ae/resource_files/02-40-1f.pdf.

Trim, J. L. M. (1980). Preface to this edition. En M. Oskarsson, Approaches to Selfassessment in Foreign Language Learning (p. vii). Oxford y Nueva York: Pergamon Press.

Wilkins, D. A. (1972). Linguistics in Language Teaching. London: Edward Arnold.

\section{SOBRE LA AUTORA}

\section{Emma García Sanz}

Doctora en didáctica de ELE y su literatura por la Universidad de Gotinga, Alemania. Actualmente, es docente en la Universidad de Duisburgo-Essen. Sus áreas de docencia e investigación son la enseñanza de ELE y la didáctica de lenguas extranjeras.

Correo electrónico: emma.garcia-sanz@uni-due.de

Orcid: 0000-0003-3392-3189. 